\title{
Colon Capsule Endoscopy: Indications, Findings, and Complications - Data from a Prospective German Colon Capsule Registry Trial (DEKOR)
}

\author{
Johannes Hausmann ${ }^{1,2}$, Andrea Tal', Artur Gomer ${ }^{1}$, Michael Philipper ${ }^{3}$, Gero Moog ${ }^{4}$, Horst Hohn ${ }^{5}$, Norbert Hesselbarth ${ }^{6}$, Harald \\ Plass $^{7}$, Jörg Albert ${ }^{8}$ and Fabian Finkelmeier ${ }^{1}$ \\ ${ }^{1}$ Department of Internal Medicine 1, University Hospital Frankfurt, Frankfurt am Main, ${ }^{2}$ Department of Internal Medicine, St. \\ Vinzenz-Hospital, Hanau, ${ }^{3}$ Gastroenterologische Facharztpraxis, Düsseldorf, ${ }^{4}$ Gastroenterologische Praxis Dr. Gero Moog, Kassel, \\ ${ }^{5}$ Gastroenterologische Praxis Dr. Horst Hohn, Koblenz, ${ }^{6}$ Endoskopiezentrum Dr. Norbert Hesselbarth, Schwalmstadt, ${ }^{7}$ Centrum für \\ ambulante Gastroenterologie, Nürnberg, ${ }^{8}$ Gastroenterologie, Hepatologie und Endokrinologie, Robert-Bosch-Krankenhaus, Stuttgart, \\ Germany
}

Background/Aims: Reliable and especially widely accepted preventive measures are crucial to further reduce the incidence of colorectal cancer (CRC). Colon capsule endoscopy (CCE) might increase the screening numbers among patients unable or unwilling to undergo conventional colonoscopy. This registry trial aimed to document and determine the CCE indications, findings, complications, and adverse events in outpatient practices and clinics throughout Germany.

Methods: Patients undergoing CCE between 2010 and 2015 were enrolled in this prospective multicenter registry trial at six German centers. Patient demographics, outcomes, and complications were evaluated.

Results: A total of 161 patients were included. Of the CCE evaluations, 111 (68.9\%) were considered successful. Pathological findings in the colon $(n=92,60.1 \%)$ and in the remaining gastrointestinal tract $(n=38,24.8 \%)$ were recorded. The main finding was the presence of polyps $(n=52,32.3 \%)$. Furthermore, five carcinomas (3.1\%) were detected and histologically confirmed later. Adequate bowel cleanliness was more likely to be achieved in the outpatient setting $(p<0.0001)$. Interestingly, 85 patients $(55.6 \%)$ chose to undergo CCE based on personal motivation.

Conclusions: CCE seems to be a reliable and safe endoscopic tool for screening for CRC and detecting other diseases. Its patient acceptance and feasibility seems to be high, especially in the outpatient setting. Clin Endosc 2021;54:92-99

Key Words: Colonic neoplasms; Control; Endoscopy; Prevention; Registries; Safety

\section{INTRODUCTION}

Despite the decreasing incidence of colorectal cancer (CRC) over the past decades, ${ }^{1,2}$ which is partly due to the implementation of screening programs, ${ }^{3} \mathrm{CRC}$ is still is one of the most

Received: February 14, 2020 Revised: April 2, 2020

Accepted: April 5, 2020

Correspondence: Fabian Finkelmeier

Department of Internal Medicine 1, University Hospital Frankfurt, Theodor-Stern-Kai 7, Frankfurt am Main 60590, Germany

Tel: +49-69-63010, Fax: +49-69-6301-83112, E-mail: Fabian.Finkelmeier@kgu.de ORCID: https://orcid.org/0000-0001-8559-9910

(c) This is an Open Access article distributed under the terms of the Creative Commons Attribution Non-Commercial License (http://creativecommons.org/ licenses/by-nc/3.0) which permits unrestricted non-commercial use, distribution, and reproduction in any medium, provided the original work is properly cited. common cancers with more than 1 million newly diagnosed cases each year. ${ }^{4}$ Despite tremendous efforts to promote CRC screening, ${ }^{5,6}$ the acceptance of conventional colonoscopy as the most sensitive screening method for CRC is still low. ${ }^{7}$ This is particularly alarming, as CRC-associated mortality could be significantly reduced by adequate and rigorous screening. ${ }^{8}$ The typical reasons for the low screening numbers are the invasive nature of colonoscopy, possible complications such as bleeding or perforations, possible need for sedation, and perception of the procedure itself being unpleasant. ${ }^{9,10}$ Hence, other reliable screening tools will be essential to increase the number of patients undergoing screening. In 2006, the first generation of colon capsule endoscopy (CCE-1) (PillCam Colon; Given Imaging Inc., Yoqneam, Israel [now Covidien/ Medtronic]), a wireless and less invasive method to visualize 
the large bowel, was introduced. ${ }^{11}$ In 2009, the second generation of colon capsule endoscopy (CCE-2) was developed, allowing for a panoramic view owing to an increased view angle and an adjustable frame rate. ${ }^{12}$ Since its development, CCE has been evaluated and established for several indications. ${ }^{13}$ Although capsule endoscopy of the small bowel quickly found its place as a first-line imaging device for patients with obscure gastrointestinal (GI) bleeding, CCE was initially met with skepticism. This was because of the high procedural costs, need for extensive bowel cleansing to gain reasonable adenoma detection rates, and limitation in taking biopsies. Therefore, an additional conventional colonoscopy to confirm findings or remove polyps is required. However, CCE seems to significantly increase screening participation when offered as an alternative to conventional colonoscopy even with the knowledge that a later colonoscopy might be necessary. ${ }^{14} \mathrm{We}$ initiated a multicenter registry trial to review the current practice of CCE in Germany.

\section{PATIENTS AND METHODS}

\section{Study design and aims}

In this prospective multicenter registry trial, CCE videos of 161 patients from six investigational centers were evaluated. Owing to the observational nature of this registry trial, not all information was available for each patient. Therefore, only 153 patients were included in the final analysis. For the eight excluded patients, the provided data were insufficient to allow for any further analysis. In reviewing current practice, we explored whether CCE is a reliable, safe, and well-tolerated method. Furthermore, we sought to determine how often a suspected disease or a pathological finding could be detected or ruled out, if and what complications may occur, and whether any differences in practice-with particular regard to bowel preparation and, consecutively, bowel cleanliness-could be observed.

\section{Patient characteristics and data collection}

A total of 161 patients at six centers (five gastroenterology outpatient practices and the University Hospital Frankfurt, Frankfurt, Germany) between 2010 and 2015 were included in this prospective study. The inclusion criteria were age $>10$ years, willingness to undergo CCE, and ability to provide informed consent. Patients underwent CCE either as an outpatient procedure (five centers) or during hospitalization (one center). Relevant patient data were collected with the help of an electronic case report file (eCRF). The eCRF documented-aside from necessary patient information-the indication for CCE, bowel preparation, results of successful and complete
CCE, pathological findings, adverse events, treatment, and diagnostic consequences as well as follow-up information. Approval was obtained from the local ethics committee of the University Hospital Frankfurt before the start of the study (file no. 265/13; National Clinical Trial identifier: NCT02182466).

\section{PillCam colon capsule endoscope CCE-2}

All patients in our study received the PillCam colon capsule endoscope CCE-2 (Given Imaging). The CCE system consists of three components: the ingestible capsule endoscope $(32.2 \times 11.6 \mathrm{~mm})$ with two head cameras with a $172^{\circ}$ view angle, the sensing system attached to the patient's abdomen, and the workstation with RAPID 8 software (Covidien/ Medtronic). The images are taken by the capsule itself and sent to a data recorder via the sensors in the sensing system. The software displays the images on a workstation for review and analysis. The battery power lasts for about 12 hours. The capsule provides feedback to the patient through vibration when the small-bowel mucosa is detected for the first time. With the help of the movement adaptive frame rate, images are taken in a slow rate of 4 pictures per second when the capsule is traveling slowly and increased to 35 pictures per second with a higher speed of the capsule.

\section{Bowel preparation and evaluation of cleanliness}

The procedure for bowel preparation was mainly based on the European Society of Gastrointestinal Endoscopy guidelines. ${ }^{13}$ In all patients, the last solid food intake was 1 or 2 days before CCE. Thereafter, a 1- or 2-day bowel cleansing protocol with polyethylene glycol (PEG) was performed. Boosting with sodium phosphate $(\mathrm{NaP})$ or ascorbic acid was allowed. The use of simethicone as an anti-foaming agent and the use of bisacodyl were also permitted. Chronic or acute kidney disease had to be ruled out before the use of $\mathrm{NaP}$. The level of bowel cleanliness was initially assessed by applying the fourpoint grading scale (poor, fair, good, and excellent) suggested by Leighton et al. ${ }^{15}$ For simplification, the four-point grading scale of cleanliness was shortened to a two-point grading scale, in which poor and fair results were deemed "inadequate", whereas good and excellent results were considered "adequate".

\section{Quality of CCE videos and analysis}

All videos were analyzed under standardized conditions in a closed room with elimination of disturbances during the evaluation. Highly experienced investigators conducted the CCE video analyses. The evaluation started with the identification of the first and last colonic image. A CCE was considered complete when the hemorrhoid plexus was visualized. If the CCE was incomplete, the last picture was documented with the suspected anatomical localization. The total transit time 
was measured, starting from the ingestion of the capsule until the visualization of the hemorrhoid plexus. Moreover, the colon transit time was measured from the visualization of the cecum until the hemorrhoid plexus. All pathological findings were digitally marked using the thumbnail function of RAPID 8 software. After finishing the video analysis, all findings were documented in the eCRF. A colonic polyp was considered significant if it was $6 \mathrm{~mm}$ in size.

When the CCE results were compared, the defined gold standard for colon examination was a flexible colonoscopy. Intubation of the cecum and a drawback time of at least 6 min were the defined standards for screening colonoscopy. Intubation of the terminal ileum was not mandatory and left to the discretion of the investigator when indicated (e.g., in the presence of diarrhea or chronic inflammatory bowel disease [IBD]).

The use of a patency capsule for diagnosing suspected stenosis of the small bowel before the use of CCE was based on the investigator's discretion.

\section{Statistical analysis}

This study was designed as a prospective registry trial. All patients with informed consent were included at the respective centers. The primary outcomes were the indications, findings, and complications of CCE. Continuous variables are shown as median and range, and categorical variables are reported as frequencies and percentages. To determine the impact of quantitative parameters for the defined groups, the WilcoxonMann-Whitney $U$-test was applied. Values of $p<0.05$ were considered statistically significant. Statistical analyses were performed using SPSS 22 (IBM, Armonk, NY, USA) and Prism 5 (GraphPad Software Inc., San Diego, CA, USA).

The local ethics committee of the University Hospital Frankfurt approved this study (no. 265/13).

\section{RESULTS}

\section{Study population and patient characteristics}

A total of 161 patients who underwent CCE with CCE-2 at six study centers between 2010 and 2015 were included. As this was an observational study, not all information was available for all patients. Therefore, only 153 patients were included in the final analysis; 8 patients were completely excluded because there was no information allowing for any analysis. Eighty-three patients were men (51.6\%), and the median age of the entire cohort was 57 years (range, 12-89 years). Three patients were younger than 18 years (two patients were 15 years old and one patient was 12 years old).

\section{Primary indications for endoscopic evaluation of the gastrointestinal tract}

Information about the primary indication for CCE was provided for 153 patients (95\%). Seventy-two patients (47.1\%) presented with clinical symptoms that mandated an endoscopic evaluation. Detailed information about suspicious symptoms was documented for 68 of the 72 symptomatic patients. The symptoms were abdominal pain $(n=28,41.2 \%)$, blood in stool $(n=18,26.5 \%)$, abnormal bowel movements $(n=7$, $10.3 \%)$, diarrhea or obstipation $(n=6,8.8 \%)$, anemia $(n=6$, $8.8 \%)$, and weight loss $(n=3,4.4 \%)$. Fifty-five patients (35.9\%) underwent planned CRC screening according to guidelines. Of these 55 patients, 13 (23.6\%) had an increased risk of CRC owing to familial predisposition $(n=7,4.3 \%)$ or planned con-

Table 1. Patient Characteristics

\begin{tabular}{|c|c|}
\hline & $\begin{array}{c}\text { All patients } \\
n=153\end{array}$ \\
\hline Age, median, range & $57(12-89)$ \\
\hline Male sex, $n(\%)$ & $83(51.62)$ \\
\hline Indications for endoscopy & $153(100)$ \\
\hline Symptoms, $n(\%)$ & $72(47.1)$ \\
\hline CRC screening, $n(\%)$ & $55(35.9)$ \\
\hline Suspected/underlying IBD, $n(\%)$ & $11(7.2)$ \\
\hline Other, $n(\%)$ & $15(9.8)$ \\
\hline Symptoms mandating endoscopy & $68(100)$ \\
\hline Blood in stool, $n(\%)$ & $18(26.5)$ \\
\hline Abdominal pain, $n(\%)$ & $28(41.2)$ \\
\hline Abnormal bowel movements, $n(\%)$ & $7(10.3)$ \\
\hline Anemia, $n(\%)$ & $6(8.8)$ \\
\hline Weight loss, $n(\%)$ & $3(4.4)$ \\
\hline Diarrhea or obstipation, $n(\%)$ & $6(8.8)$ \\
\hline Reasons for electing CCE & $151(100)$ \\
\hline Personal motivation/demand, $n(\%)$ & $85(56.3)$ \\
\hline Incomplete prior colonoscopy, $n(\%)$ & $51(33.8)$ \\
\hline Contraindication for colonoscopy, $n(\%)$ & $4(2.6)$ \\
\hline Evaluation of remaining gastrointestinal tract, $n(\%)$ & $11(7.3)$ \\
\hline Reasons for incomplete prior endoscopy & $42(100)$ \\
\hline Anatomical reasons, $n(\%)$ & $19(45.3)$ \\
\hline Suspected benign stenosis, $n(\%)$ & $15(35.7)$ \\
\hline Difficult sedation/comorbidities, $n(\%)$ & $2(4.8)$ \\
\hline Suspected malignant stenosis, $n(\%)$ & $1(2.4)$ \\
\hline Other, $n(\%)$ & $5(11.9)$ \\
\hline
\end{tabular}


trol endoscopy after adenoma resection $(n=6,3.7 \%)$. Eleven patients (7.2\%) underwent CCE because of an underlying or suspected IBD, and, finally, 14 patients $(9.2 \%)$ needed colonoscopy for other reasons (suspected bleeding, postoperative controls, suspected endometriosis, tumor screening after deep vein thrombosis, and suspected diverticulitis). All patient characteristics are detailed in Table 1.

\section{Reasons for ultimately selecting CCE instead of flexible colonoscopy}

Information on the indication that triggered the need for CCE was provided for 151 patients. For two patients, the indication was unknown. Eighty-five patients (52.8\%) explicitly requested for a CCE instead of flexible endoscopy, and 51 patients (31.7\%) had a history of previous incomplete colonoscopy. The most common reasons for incomplete colonoscopy were suspected postoperative adhesions/anatomical reasons (i.e., after liver transplantation, ileal neobladder reconstruction in 19 patients) or suspected benign "stenosis" (mostly due to diverticulosis, previous surgery, or polyps in 15 patients). Of the patients with a somehow suspected benign "stenosis" in colonoscopy, nine patients (54\%) had incomplete CCE because of an extended transit time and early stoppage of recording due to the "stenosis", which was either a polyp, diverticulitis, or an anatomical abnormality after surgery. However, as described, only one patient had a capsule retention, which mandated further procedures.

Four patients (2.5\%) had contraindications to colonoscopy, and 11 patients (6.8\%) requested for CCE to investigate other parts of the digestive tract. If clinically indicated, patients with suspected or confirmed stenosis $(n=3,2 \%)$ underwent a patency capsule test before CCE (Table 1).

\section{Bowel preparation}

Information on the bowel cleansing procedure was provided for 149 patients (92.5\%), who reported having their last solid food at 1 day $(n=50,31.1 \%)$ or 2 days $(n=99,61.5 \%)$ before CCE.

For a total of 152 patients, information on bowel cleanliness was provided. Ninety-nine patients (61.5\%) received PEG plus ascorbic acid as the main component. With respect to the level of cleanliness in CCE, 129 patients (80.1\%) were categorized as adequate. Twenty patients (12.4\%) were rated as "inadequate", whereas three patients $(1.9 \%)$ were rated as "information not available".

\section{Success rate of CCE}

A CCE was considered successful if the entire colon could be visualized. CCE was successful in 111 patients (68.9\%). Of 153 cases with complete information, CCE was rated unsuccessful in 42 cases (26.1\%). In eight cases, no information concerning CCE success could be retrieved (no information was entered in the registry), thus precluding a retrospective evaluation. The reasons for unsuccessful CCE were mainly incomplete colon passage $(n=33,20.5 \%)$ and inadequate bowel cleansing $(n=7,4.3 \%)$. In two cases $(4.7 \%)$, recording errors due to technical malfunction of the system resulted in an unsuccessful CCE. In patients with incomplete CCE, the last anatomical localizations were provided for 30 of 42 capsules (71.4\%), and were described as the sigmoid colon ( $n=17,10.6 \%)$; small intestine $(n=3,1.9 \%)$; ascending colon ( $n=4,2.5 \%)$; descending colon $(n=3,1.9 \%)$; and transverse colon, cecum, and stomach for one capsule each. Among all CCE cases, only one capsule remained in the small intestine and had to be recovered surgically (see the "Complications" section). The median complete transit time of the capsule in patients with successful colon passage was $366 \mathrm{~min}$ (range, 12-1,118 $\mathrm{min}$ ), and the median colon transit time was 198 min (range, 5-990 min). Patients undergoing CCE in an outpatient setting had a higher tendency for a successful CCE (i.e., complete visualization of the colon; $p=0.0001$ ). For detailed information, see Table 2.

\section{Pathological findings in the colon}

Information concerning pathological findings in CCE was provided for 153 patients. In 92 CCE investigations (60.1\%), at least one new finding was documented. In 61 (39.9\%) investigations, no pathological findings were detected. Of these 61 investigations, a total of 43 patients (70.5\%) had complete colon passage, and thus pathologies of the colon could be ruled

Table 2. Colon Capsule Endoscopy Success Rate

\begin{tabular}{|c|c|c|c|c|c|c|c|}
\hline & $\begin{array}{c}\text { Entire cohort } \\
n=153\end{array}$ & $\begin{array}{c}\text { Center } 1 \\
n=8\end{array}$ & $\begin{array}{c}\text { Center } 2 \\
n=39\end{array}$ & $\begin{array}{c}\text { Center } 3 \\
n=40\end{array}$ & $\begin{array}{c}\text { Center } 4 \\
n=47\end{array}$ & $\begin{array}{c}\text { Center } 5 \\
n=4\end{array}$ & $\begin{array}{c}\text { Center } 6^{a)} \\
n=15\end{array}$ \\
\hline Complete CCE, $n(\%)$ & 111 & $3(37.5)$ & $32(82)$ & $34(85)$ & $39(83)$ & $2(50)$ & $1(6.7)$ \\
\hline Incomplete CCE, $n(\%)$ & 42 & $5(62.5)$ & $7(18)$ & $6(15)$ & $8(17)$ & $2(50)$ & $14(93.3)$ \\
\hline
\end{tabular}

CCE, colon capsule endoscopy.

${ }^{a}$ Center 6 = tertiary care hospital (inpatient CCE). 

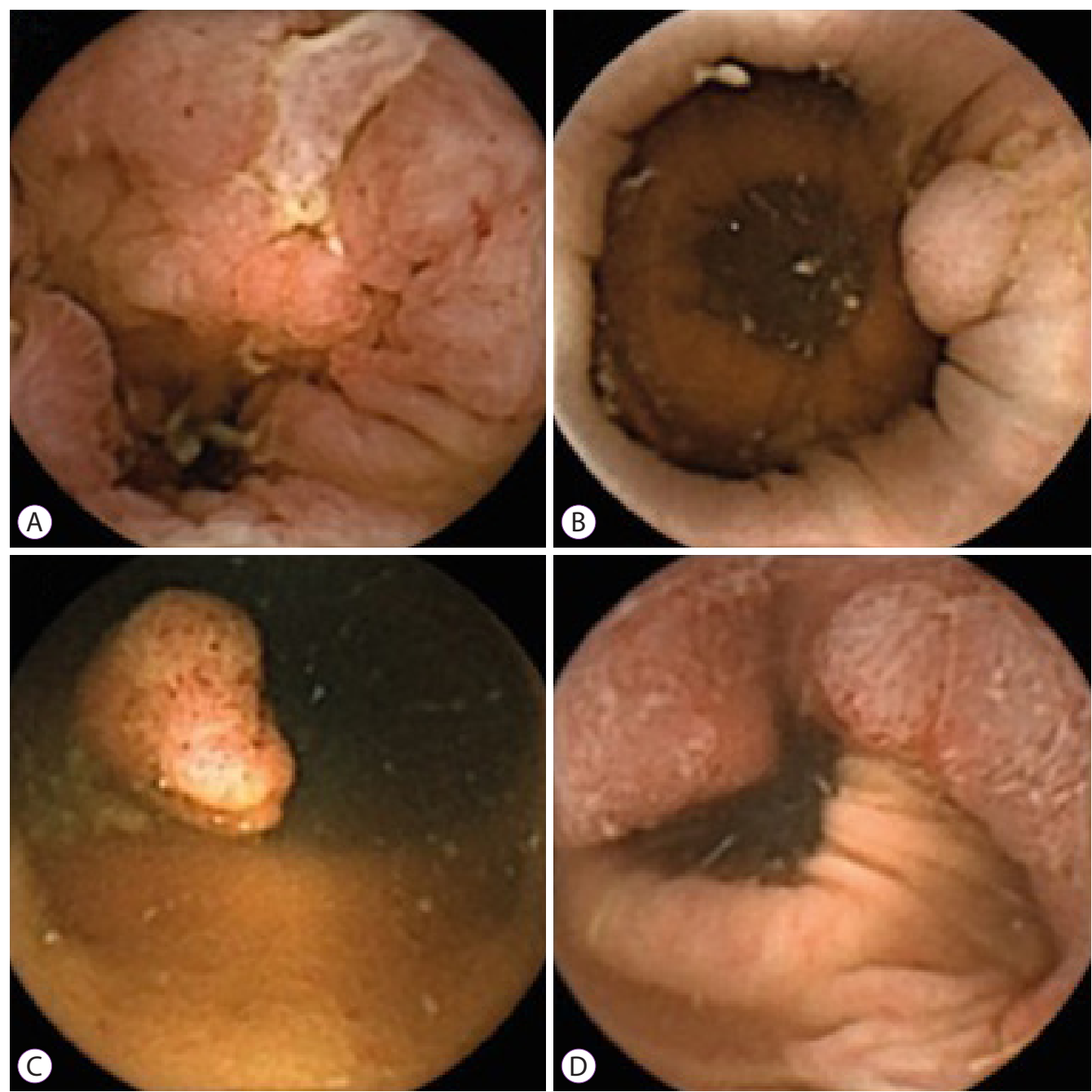

Fig. 1. Overview of the pathological findings of colon capsule endoscopy. (A) Crohn's disease of the jejunum. (B) Colon polyp. (C) Colon carcinoma. (D) Close-up view of colon carcinoma.

out. Specifications concerning pathological findings were documented for 88 of the 92 patients with colonic pathologies (95.6\%). The most common finding was the presence of polyps in 52 patients (32.3\%). In 24 patients, the detected polyps were considered significant (i.e., $6 \mathrm{~mm}$ ). In two patients (1.2\%), an adenocarcinoma was suspected in CCE. Another major finding was diverticulosis in 21 patients (13\%). Bleeding in the colon was detected in four patients (2.5\%), and other pathological findings (erosions, angiodysplasia, suspected diverticulitis) were reported for nine patients (10.2\%). For a detailed listing of the documented findings, see Table 3. Examples of pathological findings are shown in Fig. 1.

\section{Pathological findings in the remaining gastrointestinal tract}

Pathological findings in the remaining GI tract were found
Table 3. Observed Pathological Findings in the Colon in Colon Capsule Endoscopy

\begin{tabular}{lc}
\hline & $\begin{array}{c}\text { All patients } \\
\boldsymbol{n}=\mathbf{1 5 3}\end{array}$ \\
\hline Pathological findings, $n(\%)$ & $92(60.1)$ \\
Polyps, $n(\%)$ & $52(32.3)$ \\
Significant polyp (>6 mm), $n(\%)$ & $24(26.3)$ \\
\hline Suspected malignant lesions, $n(\%)$ & $2(1.2)$ \\
Diverticulosis, $n(\%)$ & $21(13)$ \\
Bleeding, $n(\%)$ & $4(2.5)$ \\
Unspecific inflammation, $n(\%)$ & $2(1.2)$ \\
Angiectasis, $n(\%)$ & $2(1.2)$ \\
Diverticulitis, $n(\%)$ & $1(0.6)$ \\
Other, $n(\%)$ & $7(7.7)$ \\
\hline
\end{tabular}


Table 4. Observed Pathological Findings in the Remaining Gastrointestinal Tract in Colon Capsule Endoscopy

\begin{tabular}{lc}
\hline & $\begin{array}{c}\text { All patients } \\
\boldsymbol{n}=\mathbf{1 5 3}\end{array}$ \\
\hline Overall pathological findings, $n$ (\%) & $38(24.8)$ \\
Esophagus, $n(\%)$ & $8(21)$ \\
Barrett's esophagus, $n(\%)$ & $4(10.5)$ \\
Other, $n(\%)$ & $4(10.5)$ \\
Stomach, $n(\%)$ & $13(34.2)$ \\
Erosion, $n(\%)$ & $3(7.9)$ \\
Gastritis, $n(\%)$ & $3(7.9)$ \\
Other, $n(\%)$ & $7(18.4)$ \\
Small intestine, $n$ (\%) & $17(44.8)$ \\
Erosion/ulcer, $n$ (\%) & $6(15.8)$ \\
Suspected Crohn's disease, $n(\%)$ & $4(10.5)$ \\
Other, $n(\%)$ & $7(18.4)$ \\
\hline
\end{tabular}

in 38 CCE cases (24.8\%). Interestingly, of the 62 patients $(40.5 \%)$ with no pathologies in the colon, 14 patients $(23 \%)$ showed extracolonic pathologies. Of the 92 patients with colonic pathologies, 22 (23.9\%) also had pathological findings outside of the colon. The most common diagnosis in the esophagus was suspected Barrett esophagus ( $n=4,2.5 \%)$, whereas 6 patients had either signs of gastritis or erosive lesions. In 17 cases (44.8\%) pathological findings in the small intestine could be detected. In four cases, Crohn's disease was suspected; other common findings were erosive lesions $(n=6)$ as well as two suspected malignancies (adenocarcinoma, neuroendocrine tumor). Rarer findings were suspected Meckel's diverticulum, scar tissue, or aphthous lesions (Table 4).

\section{Follow-up}

On the basis of the CCE findings, further diagnostic procedures were recommended in 82 patients (53.6\%). Fifty-one patients (33\%) were referred for colonoscopy, two patients (1.3\%) for a double-balloon endoscopy, and six patients (3.9\%) for a repeat procedure (including two patients with technical difficulties and four patients with initial adequate bowel cleansing). In 13 patients (8.5\%), specific medical treatment was recommended (among others, for underlying IBD). Seven patients (4.6\%) were recommended for surgical treatment. The reasons for referral were Meckel's diverticulum, abdominal adhesion, suspected colonic neoplasia, and capsule retention (in one patient) (see the "Complications" section). Three patients (1.9\%) were referred to other medical specialties. In 33 patients (21.7\%), the CCE results mandated further diagnostic or therapeutic procedures. A total of 102 patients (66.6\%) did not undergo further diagnostic procedures or treatment. Fourteen patients $(9.1 \%)$ underwent polypectomy via flexible colonoscopy. In 10 patients (6.5\%), surgery was performed. For a total of 24 patients $(15.7 \%)$, documented histology reports were available at the time of data collection, including 15 patients (9.8\%) with suspected significant polyps in CCE. Of these 15 patients, 4 (26.7\%) were diagnosed with adenocarcinoma. In the two patients in whom adenocarcinoma was suspected in $\mathrm{CCE}$, the histopathology reports confirmed the finding. Notably, in the small bowel, one adenocarcinoma and one neuroendocrine tumor was described in the histological report. In both cases, the lesions were previously suspected in CCE.

\section{Complications}

Only one major complication-capsule retention with the need for surgical retrieval-was documented in our study. The 62 -year-old female patient presented for CCE with prior incomplete colonoscopy (colonoscopy was aborted in the colon transversum owing to technical difficulty in pushing the colonoscope forward). The initial indication for endoscopy was IBD (Crohn's disease) with suspected benign stenosis in the terminal ileum. In this specific case, no patency capsule was administered before CCE. During surgery for capsule retrieval, the suspected stenosis was verified owing to IBD. No other complications were reported. All other capsules were passed naturally. Three patients of the whole cohort underwent a patency capsule test.

\section{DISCUSSION}

Over the last decade, tremendous advances have been made in the field of CCE. Primarily, polyp detection was the initial main motivation for CCE development, to provide an innovative diagnostic alternative for CRC screening and detection. Several prospective studies have compared CCE to conventional colonoscopy - the international gold standardfor the detection of significant polyps. With advances in CCE technology, recent studies were able to show comparable rates of polyp detection between CCE and flexible colonoscopy. ${ }^{12,16,17}$ However, most previous studies focused on singular aspects of CCE, such as polyp detection rate, feasibility of CCE after incomplete colonoscopy, or evaluation of bowel cleanliness. ${ }^{16,18,19}$ This study is the first to provide real-world data on CCE in Germany in a large cohort. A total of 161 patients were included, of whom 72 patients $(44.7 \%)$ presented with symptoms warranting endoscopic evaluation and 55 patients (34.1\%) needed CRC screening. Interestingly, more than half of the patients $(n=85,52.8 \%)$ chose CCE as the endoscopic procedure instead of flexible endoscopy for CRC screening, 
which underlines the previously described significant patient motivation for this relatively novel diagnostic approach. Another major reason for CCE was prior incomplete colonoscopy $(n=51,31.7 \%)$, currently one of the main indications for CCE, ${ }^{19,20}$ which strengthens its role as a supplementary endoscopic tool. ${ }^{13}$

In our study, the main reason for incomplete colonoscopy was postoperative adhesions. Successful CCE was documented in 111 patients, which translates to a success rate of $68.9 \%$. Unsuccessful CCE (42 patients, 26.1\%) was attributed to inadequate bowel cleansing in only seven patients (4.3\%). Bowel preparation and investigator-associated differences in the evaluation of bowel cleanliness have recently been the focus of scientific discussions. ${ }^{18,21}$ Therefore, differences in bowel cleanliness evaluation and, consecutively, the CCE success rate may also reflect the practice of the respective center. Our data showed a trend that patients undergoing CCE in an outpatient setting were more likely to have adequate bowel preparation and successful CCE than those undergoing CCE in hospitals. This could be because fitter patients are more likely to adhere to the more rigorous bowel cleansing protocols needed for CCE than for flexible colonoscopy.

The main pathological findings in the colon were polyps. Of 52 patients with detected colonic polyps, 24 had polyps that were considered significant (i.e., $6 \mathrm{~mm}$ ) and thus needed further endoscopic evaluation. In two patients, suspected adenocarcinoma was confirmed, and two additional incidences of adenocarcinoma in the colon were detected. Interestingly, of 92 patients $(60.1 \%)$ with colonic pathologies, $38(24.8 \%)$ also had pathological findings in the upper GI tract. Fourteen patients (23\%) had no pathologies in the colon but showed pathologies in the remaining GI tract. This illustrates that CCE provides additional diagnostic information to that provided by conventional colonoscopy, which could be of particular significance for patients with Crohn's disease.

In comparison with other alternative methods to visualize the whole intestine, such as cross-sectional imaging techniques, CCE does not require radiation. Therefore, it might reduce radiation exposure or gadolinium-associated toxicity, and increase the patient compliance in treatment surveillance.

It can be argued that other diagnostic techniques such as computed tomographic colonography (CTC) would be the faster method of performing CRC screening if a patient is unwilling or unable to undergo flexible colonoscopy. Although it is comparable in terms of sensitivity and specificity, ${ }^{22-24} \mathrm{CCE}$ still has two major advantages, namely avoiding exposure to radiation and consequently radiation-associated risks, and can be performed by a gastroenterologist, avoiding referral to another specialty and possibly prolonging the time to diagnosis.

The disadvantages of CCE include a long evaluation time.
This issue is currently addressed by a new CCE software ${ }^{25}$ algorithm designed to aid in substantially reducing the video evaluation time in the future, thereby making same-day colonoscopy after CCE a more realistic scenario in general gastroenterology practice. ${ }^{26,27}$ Of note, one study was able to show that patients preferred to undergo CCE rather than CTC despite having to wait longer for the results. ${ }^{23}$ Another drawback of CCE may be the short-term initial cost in comparison to that of conventional flexible colonoscopy. However, studies have shown that although CCE seems to be costly in the short term, it can be considered economically sound as it recruits patients who otherwise might not take part in screening measures, ${ }^{28,29}$ thus increasing the volume of total examined patients.

The limitations of this study were the lack of data on the overall preparation and evaluation time as well as the limited follow-up duration. Therefore, it cannot be definitively determined if all suspected diagnoses were confirmed by colonoscopy, how long the preparation and evaluation took in the centers, and whether differences could be observed.

In conclusion, our study provides real-world data from a large German cohort. We were able to show that patient motivation to undergo CCE, as an alternative to flexible colonoscopy, was high, although patients with a variety of indications were included. Furthermore, the extracolonic pathological findings in a certain percentage of patients without detected pathological findings in the colon itself demonstrate the possible additional diagnostic benefit of CCE. Thus, we believe that our study highlights the relevance and feasibility of CCE as a diagnostic tool in the outpatient setting, especially when this might help increase the CRC screening numbers and provide for better adherence to diagnostic and treatment suggestions also in other GI diseases (such as IBD). The preliminary results of the ONECC trial, a large prospective trial on CCE in France, also showed that CCE seems useful for detecting lesions or other pathologies in clinical practice. ${ }^{30,31}$ Nevertheless, future research should focus on advancing CCE technology to reduce the evaluation time and to include new diagnostic and possibly treatment options in order to make it more cost-effective and more widely accepted in the outpatient setting.

\section{Conflicts of Interest}

Johannes Hausmann, Andrea Tal, Michael Philipper, Horst Hohn, Jörg Albert receive lecturer fees from Covidien/Medtronic, all outside of the submitted work. The other authors have no potential conflicts of interest.

Funding

None.

Author Contributions

Conceptualization: Johannes Hausmann, Andrea Tal, Jörg Albert, Fabian 
Finkelmeier

Data curation: JH, AT, JA, FF

Formal analysis: JH, AT, Artur Gomer, JA, FF

Investigation: JH, AT, AG, Michael Philipper, Gero Moog, Horst Hohn,

Norbert Hesselbarth, Harald Plass, JA, FF

Writing-original draft: JH, AT, FF

Writing-review\&editing: JH, AT, FF

\section{ORCID}

Johannes Hausmann:

Andrea Tal:

Artur Gomer:

Michael Philipper:

Gero Moog:

Horst Hohn:

Harald Plass:

Jörg Albert:

Fabian Finkelmeier:
Norbert Hesselbarth: https://orcid.org/0000-0002-5217-2301 https://orcid.org/0000-0002-0944-229X https://orcid.org/0000-0003-3625-8903 https://orcid.org/0000-0002-9557-9662 https://orcid.org/0000-0003-0950-5737 https://orcid.org/0000-0001-7931-6910 https://orcid.org/0000-0003-1585-1708 https://orcid.org/0000-0002-0167-3077 https://orcid.org/0000-0002-3420-7371 https://orcid.org/0000-0001-8559-9910

\section{REFERENCES}

1. Welch HG, Robertson DJ. Colorectal cancer on the decline--why screening can't explain it all. N Engl J Med 2016;374:1605-1607.

2. Ryerson $A B$, Eheman $C R$, Altekruse SF, et al. Annual report to the nation on the status of cancer, 1975-2012, featuring the increasing incidence of liver cancer. Cancer 2016;122:1312-1337.

3. Holme $\varnothing$, Bretthauer M, Fretheim A, Odgaard-Jensen J, Hoff G. Flexible sigmoidoscopy versus faecal occult blood testing for colorectal cancer screening in asymptomatic individuals. Cochrane Database Syst Rev 2013;(9):CD009259.

4. Ferlay J, Soerjomataram I, Dikshit R, et al. Cancer incidence and mortality worldwide: sources, methods and major patterns in GLOBOCAN 2012. Int J Cancer 2015;136:E359-E386.

5. Skinner CS, Gupta S, Halm EA, et al. Development of the Parkland-UT Southwestern colonoscopy reporting system (CoRS) for evidence-based colon cancer surveillance recommendations. J Am Med Inform Assoc 2016;23:402-406.

6. Lee YJ, Kim ES, Choi JH, et al. Impact of reinforced education by telephone and short message service on the quality of bowel preparation: a randomized controlled study. Endoscopy 2015;47:1018-1027.

7. Siegel RL, Miller KD, Fedewa SA, et al. Colorectal cancer statistics, 2017. CA Cancer J Clin 2017;67:177-193.

8. Bujanda L, Sarasqueta C, Zubiaurre L, et al. Low adherence to colonoscopy in the screening of first-degree relatives of patients with colorectal cancer. Gut 2007;56:1714-1718.

9. Kim DH, Pickhardt PJ, Taylor AJ, et al. CT colonography versus colonoscopy for the detection of advanced neoplasia. N Engl J Med 2007;357:1403-1412.

10. Shafer LA, Walker JR, Waldman C, et al. Factors associated with anxiety about colonoscopy: the preparation, the procedure, and the anticipated findings. Dig Dis Sci 2018;63:610-618.

11. Schoofs N, Devière J, Van Gossum A. PillCam colon capsule endoscopy compared with colonoscopy for colorectal tumor diagnosis: a prospective pilot study. Endoscopy 2006;38:971-977.

12. Eliakim R, Yassin K, Niv Y, et al. Prospective multicenter performance evaluation of the second-generation colon capsule compared with colonoscopy. Endoscopy 2009;41:1026-1031.

13. Spada C, Hassan C, Galmiche JP, et al. Colon capsule endoscopy: European Society of Gastrointestinal Endoscopy (ESGE) guideline. Endosco- py 2012;44:527-536.

14. Groth S, Krause H, Behrendt R, et al. Capsule colonoscopy increases uptake of colorectal cancer screening. BMC Gastroenterol 2012;12:80.

15. Leighton JA, Rex DK. A grading scale to evaluate colon cleansing for the PillCam COLON capsule: a reliability study. Endoscopy 2011;43:123127.

16. Van Gossum A, Munoz-Navas M, Fernandez-Urien I, et al. Capsule endoscopy versus colonoscopy for the detection of polyps and cancer. $\mathrm{N}$ Engl J Med 2009;361:264-270.

17. Rex DK, Adler SN, Aisenberg J, et al. Accuracy of capsule colonoscopy in detecting colorectal polyps in a screening population. Gastroenterology 2015;148:948-957.e2.

18. Buijs MM, Kroijer R, Kobaek-Larsen M, et al. Intra and inter-observer agreement on polyp detection in colon capsule endoscopy evaluations. United European Gastroenterol J 2018;6:1563-1568.

19. Triantafyllou K, Viazis N, Tsibouris P, et al. Colon capsule endoscopy is feasible to perform after incomplete colonoscopy and guides further workup in clinical practice. Gastrointest Endosc 2014;79:307-316.

20. Alarcón-Fernández O, Ramos L, Adrián-de-Ganzo Z, et al. Effects of colon capsule endoscopy on medical decision making in patients with incomplete colonoscopies. Clin Gastroenterol Hepatol 2013;11:534-540. el.

21. Buijs MM, Ramezani MH, Herp J, et al. Assessment of bowel cleansing quality in colon capsule endoscopy using machine learning: a pilot study. Endosc Int Open 2018;6:E1044-E1050.

22. Spada C, Hassan C, Barbaro B, et al. Colon capsule versus CT colonography in patients with incomplete colonoscopy: a prospective, comparative trial. Gut 2015;64:272-281.

23. Rondonotti E, Borghi C, Mandelli G, et al. Accuracy of capsule colonoscopy and computed tomographic colonography in individuals with positive results from the fecal occult blood test. Clin Gastroenterol Hepatol 2014;12:1303-1310.

24. Pioche M, Ganne C, Gincul R, et al. Colon capsule versus computed tomography colonography for colorectal cancer screening in patients with positive fecal occult blood test who refuse colonoscopy: a randomized trial. Endoscopy 2018;50:761-769.

25. Farnbacher MJ, Krause HH, Hagel AF, Raithel M, Neurath MF, Schneider T. QuickView video preview software of colon capsule endoscopy: reliability in presenting colorectal polyps as compared to normal mode reading. Scand J Gastroenterol 2014;49:339-346.

26. Kobaek-Larsen M, Kroijer R, Dyrvig AK, et al. Back-to-back colon capsule endoscopy and optical colonoscopy in colorectal cancer screening individuals. Colorectal Dis 2018;20:479-485.

27. Hussey M, Holleran G, Stack R, Moran N, Tersaruolo C, McNamara D. Same-day colon capsule endoscopy is a viable means to assess unexplored colonic segments after incomplete colonoscopy in selected patients. United European Gastroenterol J 2018;6:1556-1562.

28. Adrián-de-Ganzo Z, Alarcón-Fernández O, Ramos L, et al. Uptake of colon capsule endoscopy vs colonoscopy for screening relatives of patients with colorectal cancer. Clin Gastroenterol Hepatol 2015;13:22932301.el.

29. Parodi A, Vanbiervliet G, Hassan C, et al. Colon capsule endoscopy to screen for colorectal neoplasia in those with family histories of colorectal cancer. Gastrointest Endosc 2018;87:695-704.

30. de Leusse A, Saurin JC. L'Observatoire national de l'endoscopie colique par capsule (ONECC). Présentation et bilan à deux ans. Acta Endoscopica 2014;44:258-261.

31. Christophe Saurin J, Ponchon T, Gay G, Vahedi K, Christophe Letard J, Benamouzig R. French muticentric experience of colon capsule endoscopy in real practice: primary results of the colon capsule endoscopy observatory "ONECC". Gastrointest Endosc 2013;77(5 Suppl):AB496-AB497. 